\title{
Dust in 3CR radio galaxies: On the FR 1-FR 2 difference ${ }^{\star}$
}

\author{
S. A. H. Müller ${ }^{1}$, M. Haas ${ }^{1}$, R. Siebenmorgen ${ }^{2}$, U. Klaas ${ }^{3}$, K. Meisenheimer ${ }^{3}$, R. Chini ${ }^{1}$, and M. Albrecht ${ }^{4}$ \\ 1 Astronomisches Institut, Ruhr-Universität Bochum (AIRUB), Universitätsstr. 150 / NA7, 44780 Bochum, Germany \\ e-mail: smueller@astro.rub.de \\ ${ }^{2}$ European Southern Observatory (ESO), Karl-Schwarzschild-Str. 2, 85748 Garching, Germany \\ 3 Max-Planck-Institut für Astronomie (MPIA), Königstuhl 17, 69117 Heidelberg, Germany \\ ${ }^{4}$ Instituto de Astronomía, Universidad Católica del Norte, Avenida Angamos 0610, Antofagasta, Chile
}

Received 6 August 2004 / Accepted 6 September 2004

\begin{abstract}
We compare three 3CR samples of 11 FR 1 galaxies, 17 FR 2 galaxies and 18 lobe-dominated quasars contained in the ISO Data Archive. In contrast to the powerful FR 2 galaxies with edge-brightened lobes, the low radio power FR 1 galaxies in our sample do not exhibit any high MIR or FIR dust luminosity, which is typical for a buried, intrinsically more luminous AGN. This consolidates the fact already inferred from optical studies that their AGNs have only a relatively low luminosity. Also the FR 1 galaxies show a high FIR/MIR luminosity ratio, compared to quasars, suggesting that their FIR luminosity is substantially powered by the interstellar radiation field (ISRF) of the giant elliptical hosts. Finally, we discuss the FR 1 -FR 2 morphological dichotomy. FR 1 galaxies do not have more interstellar matter (ISM) than FR 2s as traced - on the large scale by the cool FIR emitting dust and - in the nuclear region - by the warm MIR emitting dust. Due to the lack of central gas we suggest that the black holes of our FR 1 galaxies are fed at a lower accretion rate than those of the FR 2 galaxies.
\end{abstract}

Key words. galaxies: fundamental parameters - galaxies: photometry - quasars: general - infrared: galaxies

\section{Introduction}

Extended radio galaxies are subdivided into two classes according to their radio morphology (Fanaroff \& Riley 1974): While the FR 2 radio galaxies show powerful edge-brightened double lobes extending far beyond the host galaxy, the FR 1 galaxies, exhibiting also lower luminosities, do not. This FR 1 -FR 2 dichotomy occurs around a $408 \mathrm{MHz}$ luminosity threshold of about $10^{24.5} \mathrm{~W} \mathrm{~Hz}^{-1}$, which rises with the optical luminosity of the host galaxy (Owen \& Ledlow 1994). It is still under debate (e.g. Gopal-Krishna \& Wiita 2001), whether the dichotomy is due to intrinsic properties of the AGN launching two different types of jets or due to extrinsic properties with similar jet types but different interaction with the host galaxy or the circumgalactic environment.

The 3CR Catalogue of radio sources at $178 \mathrm{MHz}$ (Spinrad et al. 1985) contains 298 sources, with about 200 belonging to the FR 1 and FR 2 classes. The brightness of the sources makes the $3 \mathrm{CR}$ catalogue a well suited database for studying the relationship between these AGN classes.

Optical studies of the central regions provide good spatial resolution, but they may be hampered by dust extinction. In fact, HST images of 3CR radio galaxies show filamentary and clumpy dust absorption features (e.g. de Koff et al. 2000). If

^ Based on observations with the Infrared Space Observatory ISO, an ESA project with instruments funded by ESA Member States (especially the PI countries: France, Germany, the Netherlands and the UK) and with the participation of ISAS and NASA. the extinction is higher in FR 2s than in FR 1s, then observed differences of optical quantities like those found by Baum et al. (1995) may be simply produced by extinction. Furthermore, often the entire amount of dust is underestimated, when derived from optical extinction. Therefore, mid- and far-infrared (MIR, FIR) observations of dust emission are of great value.

Based on a few sparse IRAS detections and mainly upper limit constraints Golombek et al. (1988) already noted that the most powerful $3 \mathrm{CR}$ radio galaxies appear to be much more FIR luminous than the low power ones and that this could be related to the FR 1 -FR 2 dichotomy. From a statistical analysis of coadded IRAS photometry of 3CR and B2 radio galaxies, Impey \& Gregorini (1993) concluded that the more powerful and FR 2 dominated 3CR sample has warmer FIR dust emission than the less powerful and FR 1 dominated B2 sample.

With the Infrared Space Observatory ISO (Kessler et al. 2003) about 100 3CR sources of various radio-loud AGN types were observed with a detection rate above $50 \%$. The full census of the ISO 3CR observations has been published by Siebenmorgen et al. (2004) focussing on the ISOCAM MIR data and the comparison with dust models, and by Haas et al. (2004) focussing on the ISOPHOT MIR-FIR data and the orientation-dependent unification of FR 2 radio galaxies and quasars. Corroborating earlier ISO results by Meisenheimer et al. (2001), clear evidence was found that the powerful FR 2 galaxies contain a highly dust-enshrouded quasar-like AGN.

The ISO archive contains 11 3CR sources of FR 1 type with giant elliptical hosts. Here we consider what their detailed MIR 
Table 1. Parameters of the FR 1 galaxies, derived in the same way as by Haas et al. (2004). $L_{\mathrm{eml}}=L\left(\mathrm{H}_{\alpha}+[\mathrm{NII}]\right)$ is adopted from Zirbel \& Baum (1995).

\begin{tabular}{rrrrrrrrr}
\hline \hline $3 \mathrm{CR}$ & $\begin{array}{r}D_{L} \\
\mathrm{Mpc}\end{array}$ & $\begin{array}{r}L_{\mathrm{MIR}} \\
\log \left[L_{\odot}\right]\end{array}$ & $\begin{array}{r}L_{\mathrm{FIR}} \\
\log \left[L_{\odot}\right]\end{array}$ & $\begin{array}{r}P_{15 \mu \mathrm{m}} \\
\log [W]\end{array}$ & $\begin{array}{r}P_{178 \mathrm{MHz}} \\
\log [W]\end{array}$ & $\begin{array}{r}T_{\text {dust }} \\
{[\mathrm{K}]}\end{array}$ & $\begin{array}{r}M_{\text {dust }} \\
\log \left[M_{\odot}\right]\end{array}$ & $\begin{array}{r}L_{\text {eml }} \\
\log [W]\end{array}$ \\
\hline 31.0 & 70 & $<9.23$ & $<9.84$ & $<35.55$ & 33.25 & $\sim 24$ & $\sim 7.00$ & $\ldots$ \\
$66 \mathrm{~B}$ & 91 & $<8.85$ & $<9.47$ & $<35.10$ & 33.64 & $\sim 29$ & $\sim 6.23$ & $\ldots$ \\
76.1 & 136 & $<9.21$ & $<9.97$ & $<35.35$ & 33.70 & $\sim 28$ & $\sim 6.82$ & $\ldots$ \\
84.0 & 74 & 0.87 & 10.80 & 37.11 & 33.85 & 35 & 7.01 & 34.91 \\
270.0 & 31 & 8.84 & 9.05 & 35.10 & 33.03 & 28 & 5.81 & 32.76 \\
272.1 & 12 & 8.13 & 8.33 & 34.46 & 31.90 & 27 & 5.13 & 32.37 \\
274.0 & 16 & 8.43 & 8.50 & 34.72 & 32.56 & 35 & 4.75 & 33.47 \\
293.0 & 197 & 9.98 & 10.43 & 36.32 & 34.04 & 26 & 7.41 & 34.55 \\
296.0 & 104 & $<9.24$ & $<9.64$ & $<35.57$ & 33.45 & $\sim 27$ & $\sim 6.51$ & $\ldots$ \\
305.0 & 179 & 9.97 & 10.23 & 36.29 & 34.04 & 32 & 6.66 & 33.12 \\
338.0 & 123 & 9.29 & $<10.02$ & 35.52 & 34.21 & 27 & 6.95 & 33.77 \\
\hline
\end{tabular}

and FIR SEDs contribute to the current picture on the relation between FR 1 and FR 2 galaxies. We adopt a $\Lambda$ cosmology with $H_{0}=71 \mathrm{~km} \mathrm{~s}^{-1} \mathrm{Mpc}^{-1}, \Omega_{\mathrm{m}}=0.27$ and $\Omega_{\Lambda}=0.73$.

\section{Data and results}

The data are described in Siebenmorgen et al. (2004) and Haas et al. (2004). They refer to the entire 3CR observations performed by ISO. Here we use the randomly collected sample of 11 FR 1 galaxies, four observed in the MIR-FIR by ISOPHOT, and seven observed in the MIR by ISOCAM with three of these detected in the FIR by IRAS. The aperture size covers the entire galaxies, being about $30^{\prime \prime}$ at $\lambda \lesssim 25 \mu \mathrm{m}, 45^{\prime \prime}$ at $60-100 \mu \mathrm{m}$ and $90^{\prime \prime}$ at $120-200 \mu \mathrm{m}$. In the following we call the $10-40 \mu \mathrm{m}$ range MIR and the $40-1000 \mu \mathrm{m}$ one FIR.

Figure 1 shows the SEDs of all 11 FR 1 sources. The most remarkable SED features are (1) the stellar optical-NIR bump due to the elliptical hosts; (2) the well discernible dust emission bump in the MIR-FIR wavelength range; and (3) the steep $(\alpha>0.5)$ synchrotron spectrum in the $\mathrm{cm}-\mathrm{mm}$ wavelength range. The SEDs show two minima, one around $1 \mathrm{~mm}$, where the synchrotron radiation falls below the FIR dust emission, and one at $\lambda \approx 10 \mu \mathrm{m}$, where the Rayleigh-Jeans tail of the stellar bump meets the MIR-FIR emission bump. The $\lambda^{-2}$ modified greybodies refer to the cool dust component which peaks in the FIR around $100 \mu \mathrm{m}$. Their temperatures are used to derive the dust mass. Table 1 lists the luminosities and other values derived in the same manner as described by Haas et al. (2004).

For comparison we use the 3CR samples of 17 FR 2 galaxies and 18 quasars (including broad line radio galaxies) with clear thermal dust bumps (Fig. 1 and Table 2 in Haas et al. 2004).

\section{Discussion}

The strength of the AGN in FR 1s is believed to be low. HST images show only marginal evidence for thick obscuring dust tori in FR 1s, but optical observations may have missed the obscuring material. Therefore, we perform a check in the IR.

The FR 1s exhibit rather weak MIR and FIR luminosities below $10^{10} L_{\odot}$ (Table 1 ). If the FR 1 sources had powerful AGN, one should see their signatures either directly via the broad line regions or via the reemission from absorbing dust.

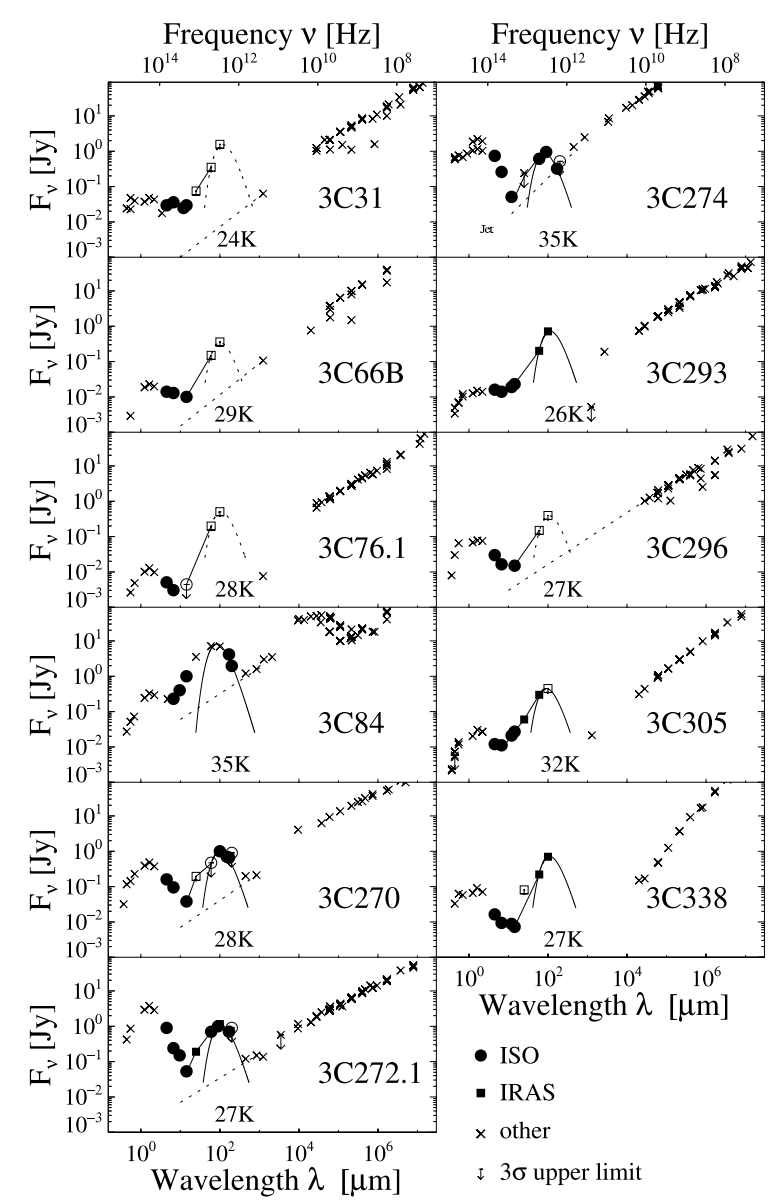

Fig. 1. SEDs of FR 1 galaxies. The measurement errors $(\$ 30 \%)$ are smaller than the symbol size. The greybody is interactively fitted to the FIR bump (solid line for detections, dotted for upper limits). The straight lines connecting the MIR and FIR data points indicate the SED envelopes from which the luminosities are derived. The straight dashed lines indicate the extrapolation of the synchrotron contribution from the $\mathrm{cm}-\mathrm{mm}$ range to shorter wavelengths, if not constrained to low values by the mm data points; for 3C 274 the dashed line shows the contribution of the jet as resolved at 12 and $450 \mu \mathrm{m}$. We have reexamined the upper limits at 6.7 and $14.3 \mu \mathrm{m}$ (Siebenmorgen et al. 2004), finding clear detections $F_{6.7 \mu \mathrm{m}}=11.1 \pm 2.7 \mathrm{mJy}$ for $3 \mathrm{C} 305$, and $F_{14.3 \mu \mathrm{m}}=10.0 \pm 3.0 \mathrm{mJy}$ for 3C 66B. 3C 338 has two small companions nearby, which are excluded in the ISOCAM photometry but included in the IRAS beam leading to a high $L_{\mathrm{FIR}} / L_{\mathrm{MIR}}$ in Fig. 3.

But they neither exhibit a MIR luminosity which would be typical for a buried powerful AGN $\left(L_{\mathrm{MIR}} \gtrsim 10^{11} L_{\odot}\right)$, nor do they show any prominent optical broad line region signatures. This confirms the known conclusion (Baum et al. 1995; Whysong $\&$ Antonucci 2004) that the low luminosity 3CR FR 1 galaxies contain only a low luminosity AGN.

The FIR luminosity of the detected FR $1 \mathrm{~s}$ is probably dominated by dust emission, since the extrapolation of the extended synchrotron spectrum reaches at most $10 \%$ of the FIR fluxes. Even in the case of a flatter nuclear synchrotron spectrum its contribution to the FIR flux peak might be below $50 \%$. However, the MIR luminosity of FR 1s may be due to three components, namely the stellar hosts, synchrotron radiation and dust emission. The latter one may be powered by the AGN, starbursts and the interstellar radiation field (ISRF). In the 


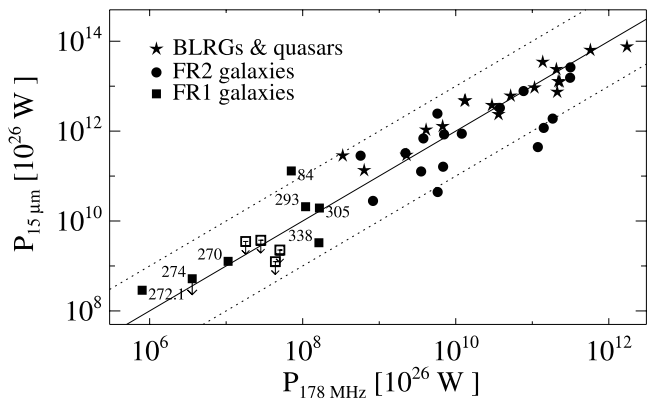

Fig. 2. MIR $15 \mu \mathrm{m}$ versus $178 \mathrm{MHz}$ radio power. Open symbols with arrows denote $3 \sigma$ upper limits. The lines indicate a ratio of 100 (solid line) and a factor of ten around this ratio (dotted lines).

following we consider, how these contributions can be discriminated by means of integral photometry.

At wavelengths $\lambda>10 \mu \mathrm{m}$ the stellar blackbody steeply declines below the observed MIR fluxes (Fig. 1) contributing at most a few percent to the entire MIR luminosities. For the two sources 3C 270 and 3C 274, which are well resolved by ISOCAM, the spatial extent $(F W H M)$ of the $14.3 \mu \mathrm{m}$ and $12.0 \mu \mathrm{m}$ emission is by a factor of two smaller than that at $4.5 \mu \mathrm{m}$. These two facts argue against a significant stellar contribution to the MIR luminosity.

The synchrotron contribution, extrapolated from the cm$\mathrm{mm}$ range (Fig. 1), lies in most cases below $30 \%$ of the MIR fluxes. An exception is the extreme case of 3C 274 (M 87) with a prominent extended optical jet. The resolved contribution of the jet reaches about $30 \%$ and $70 \%$ of the flux at $12 \mu \mathrm{m}$ and $450 \mu \mathrm{m}$, respectively (Fig. B1 in Siebenmorgen et al. 2004; Sect. 3.2 in Haas et al. 2004). In their case study of M 87 Whysong \& Antonucci (2004) find an unresolved nucleus with a $11.7 \mu \mathrm{m}$ flux density of $13 \mathrm{mJy}$ inside a synthetic $0 . ' 6$ aperture. This is consistent with our result of $35 \mathrm{mJy}$ at $12 \mu \mathrm{m}$ over a larger area $\left(\sim 20^{\prime \prime}\right)$, excluding the resolved jet flux (16 mJy). The remaining $12 \mu \mathrm{m}$ flux of $22 \mathrm{mJy}$ comes from a larger nonjet area, hence has no synchrotron origin, but rather originates from a stellar host or dust emission.

The case of M 87 demonstrates that our MIR luminosities may overestimate the MIR dust luminosity in general. For the other sources of our sample no MIR information of high spatial resolution is available in the literature. From our integral photometry we cannot properly disentangle the synchrotron and dust contributions to the MIR luminosity. We continue the discussion under the assumption that the major fraction of the MIR luminosity of most of our sources is due to dust emission.

Since the FR 1s show little evidence for luminous dust tori and their AGNs are weak, we check whether their MIR emission - if due to dust - is powered by the AGNs or by the presumably strong interstellar radiation field (ISRF) of the giant hosts. Starbursts might play only a minor role, as shown further below. Therefore we compare the MIR luminosity with two independent AGN luminosity measures:

1) The ratio of $15 \mu \mathrm{m}$ to $178 \mathrm{MHz}$ power shows the same dependency for FR 1s as for FR 2s and quasars (Fig. 2). In the FR $2 s$ and the quasars the MIR power is dominated by AGN heated dust emission (Haas et al. 2004). Even, if for

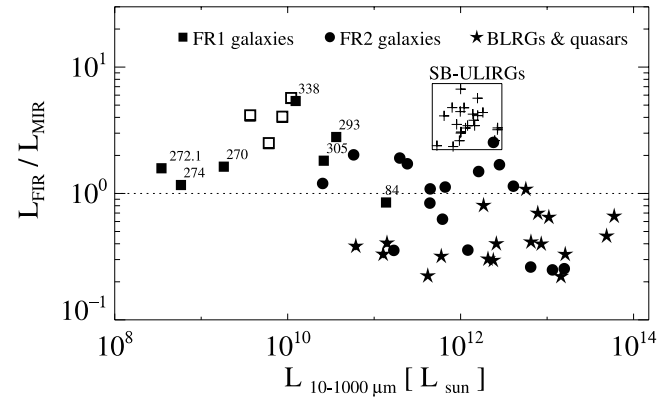

Fig. 3. Ratio of FIR to MIR versus IR luminosity. Open squares are upper MIR and FIR flux limits. The rectangular box contains the starburst ULIRGs (+) (Klaas et al. 2001). The dotted line indicates the $L_{\mathrm{FIR}} / L_{\mathrm{MIR}}$ transition border between FR 1 galaxies and quasars.

the FR 1s only $30-50 \%$ of the MIR power were due to dust, the ratio $P_{15 \mu} / P_{178 \mathrm{MHz}}$ would still lie within the dispersion of the distribution of a factor of ten. The similarity of this ratio, independent of radio power and source type, would be difficult to understand, if in the FR 1s the MIR dust emission were heated only by the ISRF without any significant AGN contribution.

2) We consider the luminosity of the narrow $\mathrm{H}_{\alpha}+[\mathrm{NII}]$ emission lines (from Zirbel \& Baum 1995) which refers to the central $2.5 \mathrm{kpc}$ of the sources. The emission line luminosity of FR $1 \mathrm{~s}$, normalized by the absolute $V$ magnitude of the host, lies between the high range of FR $2 \mathrm{~s}$ and the low one of radio-quiet elliptical galaxies (Fig. 2 in Baum et al. 1995). This argues against a pure host galaxy origin of the emission lines and favours a significant AGN contribution to the luminosity in FR 2 s and also in FR 1s. Now considering our data, the ratio of emission line luminosity and MIR $15 \mu \mathrm{m}$ power is similar for all source types (range $10^{-1}$ to $10^{-3}$ ) and independent of radio power. This would be difficult to understand if $P_{15} \mu \mathrm{m}$ were purely due to ISRF heated dust. If the emission line luminosity were significantly driven by the AGN, then $P_{15} \mu \mathrm{m}$ should include power from the AGN heated dust, too.

These two results are consistent with the interpretation that also in the FR 1s the MIR dust emission can be powered by the AGN, but a considerable contribution from the host's interstellar radiation field cannot be excluded.

As mentioned above, the FIR luminosity of FR 1s is probably dominated by dust emission. Relative to both the MIR and the radio $178 \mathrm{MHz}$ luminosities, the FIR luminosity of the FR 1 galaxies is higher than that of the quasars and many FR 2 galaxies (Fig. 3). This suggests that in the FR 1s the FIR luminosity is substantially provided by another heating source than by a weak AGN, e.g. at a first glance by starbursts. But the temperature $\mathrm{T}$ of the dust component associated with the FIR flux peak lies between $25 \mathrm{~K}$ and $35 \mathrm{~K}$ for the FR $1 \mathrm{~s}$ (Fig. 1). This is exactly the range found for radio-quiet elliptical galaxies (e.g. Bregman et al. 1998), and lower than for FR 2 s and luminous starburst galaxies $(T>35 \mathrm{~K})$. Also, the ratio of total optical luminosity, including that from the host galaxy, to the FIR luminosity is about 50-500 times higher for FR 1s than for FR 2s and dusty starburst galaxies. Therefore, 
we conclude that in FR 1s the bulk of the FIR emission is mainly powered by the ISRF of the giant host galaxy. If the dust is distributed quite homogeneously, then the ISRF may heat it to higher temperatures than those around $T=15-20 \mathrm{~K}$ found in spiral galaxies, where the bulk of the dust is typically concentrated in dense clumps shielding against the ISRF. The AGN or starbursts, if any, play only a minor role for the FIR luminosity of FR 1s. This is consistent with the results by Heckman et al. (1986) and de Koff et al. (2000) that many FR 2 hosts show morphological distortions and signs of interaction which trigger starbursts and nuclear activity, while the FR 1 hosts are typically regular ellipticals.

Finally, in the case of rather homogeneously distributed dust as indicated for the FR 1s one would expect that there is not much cold dust, which would radiate at submillimetre wavelengths and could have escaped our detection.

In order to explain why the FR 1 s do not show the powerful extended radio lobes like FR 2s, deceleration of the FR 1 jets due to entrainment of the ISM or stellar wind material has been proposed (de Young 1993; Bicknell 1994, 1995; Bowman et al. 1996). Also the existence of sources with hybrid morphology - FR 1 on one side, FR 2 on the other side of the core - argues against intrinsic fundamental physical differences in the central engine, such as black hole spin (slow for FR 1, fast for FR 2), or jet plasma composition $\left(\mathrm{e}^{-} \mathrm{p}^{+}\right.$for FR $1, \mathrm{e}^{-} \mathrm{e}^{+}$for FR 2), and favours an extrinsic origin for the FR $1-$ FR 2 dichotomy (Gopal-Krishna \& Wiita 2000). In addition to entrainment, the pressure of the external hot X-ray emitting gas turns out to play a role in the confinement of the jets (e.g. Laing \& Bridle 2002).

Since the edge-darkened FR 1 lobes are contained within the host galaxy, the relevant jet deceleration must take place therein and not far outside in the circumgalactic medium. But what is the main source for entrainment in FR 1s?

With dust masses between $10^{5}$ and $10^{7} M_{\odot}$ the FR $1 \mathrm{~s}$ (Table 1) do not show evidence for more interstellar matter (ISM) than the FR $2 \mathrm{~s}$ having dust masses between $10^{6}$ and $10^{9} M_{\odot}$ (Table 2 in Haas et al. 2004). If the FR $1-$ FR 2 dichotomy were purely due to deceleration of the jets by ISM entrainment, then one would expect the FR 1s to be less affected, contrary to what is observed. As one possible way out, the ISM in FR $2 s$ has to be concentrated in a special geometry, e.g. avoiding a bi-cone, so that the jets are not so much affected. Such an explanation, however, is questionable, since on HST images FR 2s show more disturbed dust structures (de Koff et al. 2000), while FR 1s appear more regular. Thus, that kind of presumably cool ISM, which we can infer via the dust emission, appears not to play a role for the jet entrainment.

Current models have considered stellar wind material to be entrained. Such material, even if dusty, may be too marginal to show up in our SEDs, which are dominated by the entire dust content. In addition to stellar wind material in the giant elliptical hosts of FR 1 galaxies the stars themselves crossing the jet stream could contribute considerably to the material entrained. As a rough estimate, assuming a jet opening angle of $1^{\circ}$ and that the $10^{12}$ stars of a giant elliptical galaxy are evenly distributed over the sphere, about $10^{7-8}$ stars lie on the jet trajectory, providing plenty of material, of the order of $10^{7-8} M_{\odot}$, for entrainment. Then, also the Owen-Ledlow effect can naturally be understood: more luminous hosts contain more stars and have a larger velocity dispersion; therefore, the chance that stars cross the jet stream and decelerate the jet increases with host luminosity. So far, however, it is not yet clear, what happens with a star approaching the jet stream and whether a star would survive such an event (e.g. Bednarek \& Protheroe 1997).

Furthermore, if the dust traces the amount of ISM and if the warm MIR emitting dust indicates also whether there is much ISM in the central region, then, with regard to the more dusty FR 2s, the FR 1s suffer a lack of ISM fuel for the immediate accretion region. This suggests that the black holes of the FR 1s are fed at a lower accretion rate than those of the FR 2s. A similar conclusion was reached by Baum et al. (1995) from the low emission-line luminosity of FR $1 \mathrm{~s}$.

Our IR data of $3 \mathrm{CR}$ sources suggest that a combination of central as well as extended differences should be considered for explaining the FR 1-FR 2 dichotomy. Furthermore, evolutionary effects could play a role, in the sense that the FR 1 galaxies could be preferentially old AGN with starving black holes. Since none of the FR 1 galaxies shows dust properties (like masses and temperatures) comparable to those found for the FR 2s and quasars, the results from our small randomly selected samples may be valid even more generally. Clearly, the phenomena leading to the FR 1-FR 2 dichotomy are highly complex, and further data are required, before definite conclusions about these suggestions can be drawn.

Acknowledgements. We thank the referee P. Wiita for his constructive suggestions. This work was supported by the Nordrhein-Westfälische Akademie der Wissenschaften.

\section{References}

Baum, S. A., Zirbel, E. L., \& O’Dea, C. P. 1995, ApJ, 451, 88

Bednarek, W., \& Protheroe, R. J. 1997, Proc. Rel. Jets in AGNs, 318

Bicknell, G. V. 1994, ApJ, 422, 542

Bicknell G. V. 1995, ApJS, 101, 29

Bowman, M., Leahy, J. P., \& Komissarov, S. S. 1996, MNRAS, 279, 899

Bregman, J. N., Snider, B. A., Grego, L., \& Cox, C. V. 1998, ApJ, 499, 670

de Koff, S., Best, P., Baum, S., et al. 2000, ApJS, 129, 33

de Young, D. S. 1993, ApJ, 405, L207

Fanaroff, B. L., \& Riley, J. M. 1974, MNRAS, 167, 31

Golombek, D., Miley, G. K., \& Neugebauer, G. 1988, AJ, 95, 26

Gopal-Krishna, \& Wiita, P. J. 2000, A\&A, 363, 507

Gopal-Krishna, \& Wiita, P. J. 2001, ASP Conf. Ser., 250, 290

Haas, M., Müller, S. A. H., Bertoldi, F., et al. 2004, A\&A, 424, 531

Heckman, T. M., Smith, E. P., Baum, S. A., et al. 1986, ApJ, 311, 526

Impey, Ch., \& Gregorini, L. 1993, AJ, 105, 853

Kessler, M., Müller, Th., Leech, K., et al. 2003, The ISO Handbook, ESA-SP, 1262

Klaas, U., Haas, M., Müller, S. A. H., et al. 2001, A\&A, 379, 823

Laing, R. A., \& Bridle, A. H. 2002, MNRAS, 336, 1161

Meisenheimer, K., Haas, M., Müller, S., et al. 2001, A\&A, 372, 719

Owen, F., \& Ledlow, M. 1994, ASP Conf. Ser., 54, 319

Siebenmorgen, R., Freudling, W., Krugel, E., \& Haas, M. 2004, A\&A, 421,129

Spinrad, H., Djorgowski, S., Marr, J., \& Aguilar, L. 1985, PASP, 97, 932

Whysong, D., \& Antonucci, R. 2004, ApJ, 602, 116

Zirbel, E. L., \& Baum, S. A. 1995, ApJ, 448, 521 